\title{
Gingival-Derived Mesenchymal Stem Cells Protect Against Sepsis and Its Complications
}

\author{
Xishuai Wang (1D ${ }^{1,2}$ \\ Hanan Song' \\ Shiyu Zhao' \\ Weijun Guan' \\ Yang $\mathrm{Gao}^{3}$ \\ 'Department of Animal Genetic \\ Resources, Institute of Animal Science, \\ Chinese Academy of Agricultural \\ Sciences, Beijing, I00193, People's \\ Republic of China; ${ }^{2}$ College of P.E and \\ Sport, Beijing Normal University, Beijing, \\ 100875, People's Republic of China; \\ ${ }^{3}$ Institute of Physical Educational and \\ Training, Capital University of Physical \\ Education and Sport, Beijing, I00I9I, \\ People's Republic of China
}

Objective: In the present study, we separated and characterized mouse gingival-derived mesenchymal stem cells (GMSCs) and investigated whether GMSCs can improve lipopolysaccharide (LPS)-induced sepsis and its complications.

Methods: Ninety-six ICR mice were randomly divided into the following groups: the control (Sham), LPS, and LPS + MSC groups. Mice received $5 \mathrm{mg} / \mathrm{kg}$ LPS intraperitoneally to induce sepsis. Histopathological micrographs illustrated organ injury. We detected systemic inflammation, blood glucose levels, and serum levels of high-mobility group box 1 (HMGB1) and lactate. In addition, pulmonary inflammation, lung permeability, and oxidative stress-related indicators in lung tissue were measured.

Results: We successfully separated a novel population of MSCs from mouse gingiva. These cells had MSC-associated properties, such as a typical fibroblast-like morphology, multiple differentiation potential, and certain phenotypes. Cell-based therapy using GMSCs significantly improved the survival rate, systemic inflammation, hypoglycemia, multiple organ dysfunction syndrome (MODS), and aortic injury during sepsis. GMSCs administration reduced pulmonary inflammation, lung permeability, and oxidative stress injury. GMSCs administration reduced neutrophil infiltration partly because GMSCs inhibited neutrophil chemoattractants tumor necrosis factor (TNF- $\alpha$ ), C-X-C motif chemokine ligand (CXCL-1), and Interleukin (IL-8). GMSCs impaired LPS-induced HMGB1 and lactate release during sepsis.

Conclusion: GMSCs administration is a novel therapeutic strategy targeting aerobic glycolysis for the treatment of sepsis because GMSCs impair LPS-induced HMGB1 and lactate release. GMSCs alleviate lung injury partly because GMSCs exert immune effects, inhibit neutrophilic inflammation, and reduce oxidative stress injury.

Keywords: mesenchymal stem cells, sepsis, ALI, neutrophilic inflammation, oxidative stress, Warburg effect

\section{Introduction}

Sepsis, which presents many complications such as multiple organ dysfunction syndrome (MODS), pathoglycemia, and hypotension, results in a high mortality rate and stems from systemic infections involving alterations to inflammatory parameters and oxidant status in many tissues. ${ }^{1,2}$ There is no effective treatment for sepsis, and the development of effective treatments is urgently needed.

Mesenchymal stem cells (MSCs) had substantial therapeutic promise for a wide range of diseases, including sepsis and acute lung injury (ALI), owing to their low immunogenicity, potent immunomodulatory potential, and paracrine capability. ${ }^{3-6}$ The beneficial effects of MSCs administration in a sepsis model were identified. ${ }^{7-15}$
Correspondence: Weijun Guan; Yang Gao Email guanweijun1967@163.com; gaoyang195528@I26.com 
MSCs administration reduced lung injury by inhibiting the excessive inflammatory response in mice with sepsis. ${ }^{7-11}$ MSCs administration resulted in reduced kidney injury during polymicrobial sepsis. ${ }^{12}$ MSCs administration improved systemic response and myocardial function during endotoxemia. ${ }^{13,14}$ Previous studies ignored the effects of MSCs on large blood vessel injuries, such as aortic injury, in a sepsis model. Vascular injury was a critical cause of the development of ALI and sepsis. ${ }^{15,16}$ Generally, vascular injury occurred before the onset of inflammatory cell infiltration and organic damage. ${ }^{17,18}$ In addition, arterial hypotension, which can be caused by large blood vessel damage, led to organ dysfunction and septic shock, which were the most severe complications of sepsis and other related deadly diseases. ${ }^{19,20}$ We examined the effects of sepsis and GMSCs on large blood vessels.

Disordered inflammation is a crucial component in the pathogenesis of sepsis and ALI. Sepsis is characterized by an uncontrolled inflammatory response involving inflammatory factors, such as IL- $1 \beta$ and TNF- $\alpha$, and effector cells, such as neutrophils. ${ }^{21,22}$ There is convincing evidence that deleterious activation of neutrophils is a critical reason leading to host tissue injury and organ damage during sepsis. ${ }^{23}$ MSCs administration is known to reduce neutrophil content in LPS-induced ALI and sepsis. $^{7,24}$ However, the underlying mechanisms by which MSCs reduce neutrophil content in septic mice require further research.

The "Warburg effect", which was first found in cancer cells, described the phenomenon that tumor cells preferentially utilized aerobic glycolysis rather than oxidative phosphorylation for energy production: this effect was also a vital aspect of innate and adaptive immunity. ${ }^{25,26}$ There is convincing evidence that activated immune cells, including macrophages, neutrophils, and $\mathrm{T}$ cells, switch from oxidative phosphorylation to aerobic glycolysis in a manner similar to tumor cells. ${ }^{25}$ This alteration contributed to the immunoregulation capability and represented a new treatment for immune system diseases. ${ }^{26}$ LPS injection is known to induce a switch from oxidative phosphorylation to aerobic glycolysis in immune cells, including dendritic cells and macrophages. ${ }^{27}$ Increased aerobic glycolysis consumed a large amount of glucose and produced a large amount of lactate. Increased serum lactate levels were a biological marker of mortality and multiple system organ failure during sepsis, and lactate clearance was a novel therapeutic method for sepsis. ${ }^{28-30}$ In addition, lactate which is produced by aerobic glycolysis stimulates macrophages to release HMGB $1 .{ }^{31} \mathrm{HMGB} 1$ is a promising therapeutic target for sepsis treatment. ${ }^{32,33}$ The Warburg effect opened the door to developing new treatments for inflammatory diseases, including sepsis. ${ }^{26,34}$ GMSCs administration may impair LPS-induced lactate and HMGB1 release during sepsis.

Toll-like receptor 4 (TLR4) stimulated human artery endothelial cells to release proinflammatory cytokines, including IL-1 $\beta$ and TNF- $\alpha .{ }^{35,36}$ TLR4/myeloid differentiation factor 88 (MyD88) signal pathway, which can be activated by LPS, was a crucial component in the pathogenesis of sepsis. ${ }^{37}$ Activation of TLR4/MyD88 signaling was a vital component in the development of sepsis. ${ }^{38}$ LPS administration led to an excessive inflammatory response via TLR4/MyD88 signaling, GMSCs administration may improve sepsis via TLR4/MyD88 signaling.

\section{Methods}

\section{Separation and Culture of GMSCs}

Gingival tissues from the maxillary molar region were separated and washed 5-7 times with sterile PBS, and as many blood cells as possible were removed. Then, the connective tissues were carefully removed. The remaining gingival tissues were mechanically minced into tiny fragments $\left(1 \mathrm{~mm}^{3}\right)$, and the extracellular matrix of the minced tissues was dissociated with $3 \mathrm{mg} / \mathrm{mL}$ trypsin II and $2 \mathrm{mg} / \mathrm{mL}$ collagenase type I at $37^{\circ} \mathrm{C}$ for 70 minutes. A complete DMEM/F12 medium was utilized to immediately neutralize the enzymatic reaction. A $100-\mu \mathrm{m}$ mesh sieve was utilized to filter through the cell suspension. After centrifugation, the cell pellets were resuspended in a complete DMEM/F12 medium and seeded into 6-well plates and cultured in a humidified incubator with $5 \% \mathrm{CO}_{2}$. To minimize heterogeneity, gingival tissues were separated from six individual mice.

The supernatant was discarded, and the nonadherent cells and exfoliated tissue masses were carefully removed. Henceforth, the medium was replaced every 24-48 h. $0.25 \%$ trypsin/EDTA was utilized to passage the GMSCs at a 1:2 dilution.

\section{Immunofluorescence Staining}

Paraformaldehyde prefixed GMSCs were permeabilized using $0.1 \%$ Triton $\mathrm{X}-100.10 \%$ sheep serum was used to block nonspecific binding sites. After blocking, the GMSCs were incubated with the following primary antibodies: FITCrabbit Sox-2, OCT-4, CD34, CD45, CD73, CD90, and CD105. After incubation, the GMSCs were incubated in 
PBS containing FITC-conjugated goat anti-rabbit secondary antibodies, followed by $3 \times 5 \mathrm{~min}$ washes. The GMSCs were counterstained with $1 \mu \mathrm{g} / \mathrm{mL}$ DAPI. Finally, the fluorescence signals were captured under a fluorescence microscope.

\section{Multiple Differentiation Potential of GMSCs \\ Osteogenic Differentiation of GMSCs}

GMSCs were seeded in 12 -well plates $\left(2.0 \times 10^{5}\right.$ cells/ well). The medium was replaced with an osteogenic differentiation medium when the cells reached $30 \%$ confluence. The osteogenic medium was refreshed with 50\% fresh medium every 2 days. On day 21 of culture and differentiation, calcium deposition was identified by performing Alizarin Red staining. All information on the induction medium is listed in Supplementary Material 1.

\section{Adipogenic Differentiation of GMSCs}

Third-generation GMSCs were inoculated in 12-well plates $\left(2.0 \times 10^{5}\right.$ cells/well $)$. The medium was replaced with an adipogenic induction and differentiation medium when cells were $30 \%$ confluent. The adipogenic medium was refreshed with $50 \%$ fresh medium every 2 days. Afterward, Oil Red O staining was performed to visualize the accumulation of intracellular lipid droplets.

\section{Chondrogenic Differentiation of GMSCs}

For chondrogenic differentiation, third-generation GMSCs were cultured in chondrogenic medium when they proliferated to $30 \%$ confluence. The chondrogenic medium was refreshed with $50 \%$ fresh medium every 2 days. On day 21 of culture, Alcian Blue staining was performed to confirm chondrogenic differentiation.

\section{Neuroblastic Differentiation of GMSCs}

When $30 \%$ confluence was achieved, GMSCs were exposed to a neuroblastic differentiation medium, which was replaced with $50 \%$ fresh medium every 2 days. On day 21 of differentiation and culture, the cellular morphology of GMSCs was detected.

\section{Experimental Groups and LPS-Induced Septic Model}

All protocols used in this study were approved by the Animal Experimental Welfare of the Institute of Animal Science, Chinese Academy of Agricultural Sciences (Beijing, China). All experiments were performed in accordance with the Animal Experimental Welfare of the
Institute of Animal Science, Chinese Academy of Agricultural Sciences and the Guide for the Care and Use of Laboratory Animals published by the US National Institutes of Health. Thirty male ICR mice (8 weeks old, 18-21 g) were provided by Beijing HFK Biotechnology Co., Ltd. (Beijing, China).

The septic model was established by administering an intraperitoneal injection of $5 \mathrm{mg} / \mathrm{kg}$ LPS (O55:B5, SigmaAldrich, St. Louis, MO, USA). Ninety-six male mice were randomized into the following groups: (1) in the control group (Sham), the mice received equal volumes of normal saline intraperitoneally; (2) in the LPS group, the mice received $5 \mathrm{mg} / \mathrm{kg}$ LPS intraperitoneally; (3) in the MSCs + LPS group, the mice received $5 \mathrm{mg} / \mathrm{kg}$ LPS intraperitoneally. Two hours later, the mice received $1 \times 10^{6}$ GMSCs via tail vein injection. The mice were received anti-rejection drug cyclosporine intraperitoneally once a day for one week before the experiment. The mice received $2 \mathrm{mg} / \mathrm{kg}$ of cyclosporine intraperitoneally as previously described. ${ }^{39-41}$ The mice were killed at 6 h, 12 h, 24 h, and 48 h. Each group contained 8 mice.

\section{Collection of Tissue Samples and Bronchoalveolar Lavage Fluid (BALF)}

Blood samples from mice were harvested from the retroorbital sinus and placed into a blood collection vessel containing an anticoagulant. After centrifugation, the upper serum layer was got and frozen at $-20^{\circ} \mathrm{C}$. After sampling, the tissues were immediately transferred to liquid nitrogen tanks.

Two milliliters of ice-cold PBS was utilized for wholelung lavage, the whole BALF was flushed five times, and the output fluid was harvested. The supernatant was immediately stored at $-20^{\circ} \mathrm{C}$.

\section{Histopathology}

The lung, liver, kidney, heart, and aortic tissue samples were dehydrated and embedded in paraffin. Five- $\mu \mathrm{m}$-thick sections were stained with HE. Photographs taken using an investigator (Olympus Corp., Japan) were used to perform morphometric measurements.

\section{Pulmonary Edema}

To quantify the degree of pulmonary edema, the protein content in BALF and the wet/dry ratio (W/D) of lung tissues were detected. The lung tissues were dried until a stable dry weight was obtained. W/D = wet weight of the lung tissue/dry weight of the lung tissue. A Bio-Rad 
protein assay kit was utilized to calculate the protein concentration in BALF.

\section{Determination of Oxidative Stress Index} MDA, SOD, MPO, and GSH expressions in the mice were detected using spectrophotometry, as previously described following the instructions. ${ }^{54,55}$

\section{Detection of Inflammatory Factors Levels}

Levels of anti-inflammatory cytokines (IL-1RN and IL-10) and pro-inflammatory cytokines (IL-1 $\beta$ ) and neutrophil chemoattractants (CXCL-1, IL-1 $\beta$, and TNF- $\alpha$ ) in BALF and levels of CXCL-1, IL-1 $\beta$, IL-8, and TNF- $\alpha$ in serum were detected using mouse enzyme-linked immunosorbent assay (ELISA) kits.

\section{Quantitative Real-Time PCR}

Total RNA of lung tissues was extracted and used to synthesize cDNAs. Real-time fluorescence quantitative PCR was performed as described, ${ }^{27}$ Supplementary Material 2 listed the murine PCR primer sequence information.

\section{Survival Analysis}

For survival analysis, 60 male mice were divided into the following groups: Sham, LPS, and LPS + MSC. LPS mice received $12 \mathrm{mg} / \mathrm{kg}$ LPS (O55:B5, Sigma-Aldrich, St. Louis, MO, USA) intraperitoneally. Sham mice received with the equal volumes of saline. LPS + MSC mice received $12 \mathrm{mg} / \mathrm{kg}$ of LPS intraperitoneally and then received $1 \times 10^{6}$ GMSCs via a tail vein injection two hours later. All mice received anti-rejection drug cyclosporine once a day for one week before the experiment. The number of dead mice was recorded every 6 hours for 48 $\mathrm{h}$, and each group included 20 mice.

\section{Detection of the Levels of Lactate, Blood Glucose,} and HMGBI

A blood glucose meter was utilized to assess the blood glucose levels. Lactate in serum was detected with a colorimetric L-lactate assay kitactate assay kit. The serum levels of HMGB1 were measured using a mouse ELISA kit. The serum levels of HMGB1 were measured with a commercial kit (Shino Test Corporation, Tokyo, Japan $)^{27}$ The GLUT1 and HMGB1 mRNA expression levels in lung tissue were detected.

\section{Statistical Evaluation}

We computed the figures and data with either GraphPad Prism 6 software or SPSS 21.0 (SPSS Inc., Chicago, IL, USA). Statistical differences among the groups were assessed using one-way ANOVA. The significance threshold was set to $P<0.05$. All data are expressed as the mean $\pm \mathrm{SD}(\mathrm{x} \pm \mathrm{s})$.

\section{Results}

\section{Biological Characteristics of GMSCs}

The cellular morphology of the GMSCs was determined at different passages P1 (Figure 1A-a), P5 (Figure 1A-b), P15 (Figure 1A-c), and P25 (Figure 1A-d). Under osteogenic induction conditions for 21 days, many calcium nodules appeared in the cytoplasm, and Alizarin Red staining showed that the reaction of calcifying nodules was positive in differentiated GMSCs (Figure 1B-a). Under adipocytic induction conditions for 21 days, many lipid droplets formed in the cytoplasm, and lipid droplets were successfully stained with Oil Red O (Figure 1B-b). GMSCs were positive for Alcian Blue staining after incubation in a chondrogenic medium for 21 days (Figure 1Bc). Under neurogenic induction conditions for 21 days, the cellular morphology changed from a spindle-shaped morphology to a multipolar and stellate morphology, and the GMSCs grew many branches and formed many synapses on the cell surface (Figure 1B-d). The GMSCs surface antigens CD73, CD90, CD105, Sox-2, and OCT-4 were positively expressed, while CD34 and CD45 were negatively expressed, as identified by immunofluorescence staining (Figure 1C).

\section{Mortality Rate}

Sham-operated mice exhibited 100\% survival. Compared with the LPS group, GMSCs administration dramatically improved survival $(P<0.01$; Figure $2 \mathrm{~A})$.

\section{Systemic Inflammation}

In mice treated with LPS, the serum levels of neutrophil chemoattractants CXCL-1 (Figure 2B; $P<0.05$ ), TNF- $\alpha$ (Figure 2C; $P<0.05$ ), and IL-8 (Figure 2D; $P<0.05$ ) and proinflammatory cytokine IL-1 $\beta$ (Figure 2E; $P<0.05$ ), upregulated within 6 hours, downregulated at 12 hours, and downregulated again at 24 hours. GMSCs administration prevented the upregulation of proinflammatory cytokine concentration IL-1 $\beta \quad(P<0.01)$. Cytokine concentrations of neutrophil chemoattractants IL-8 $(P<$ 

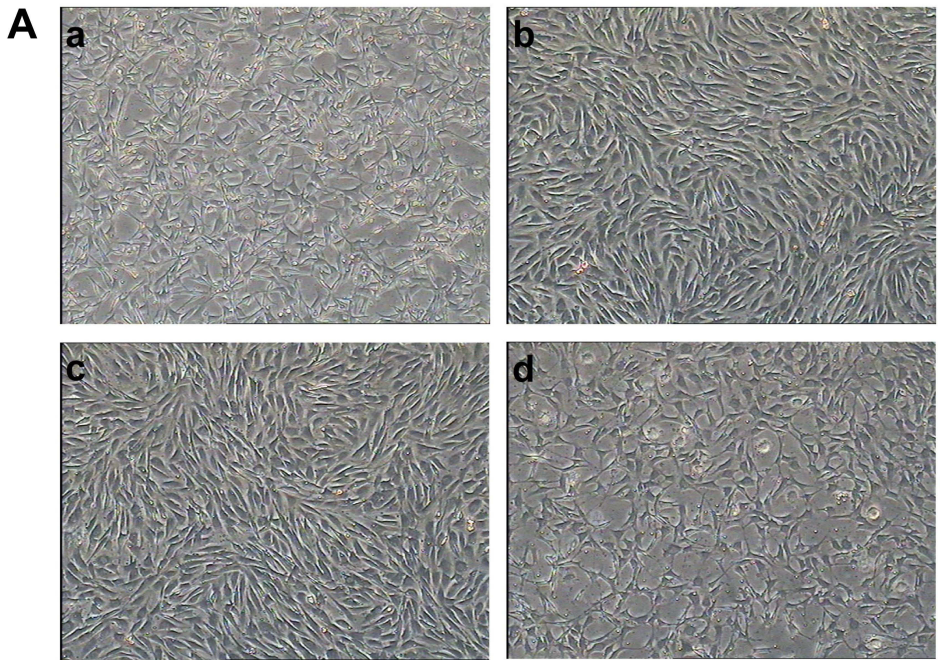

B
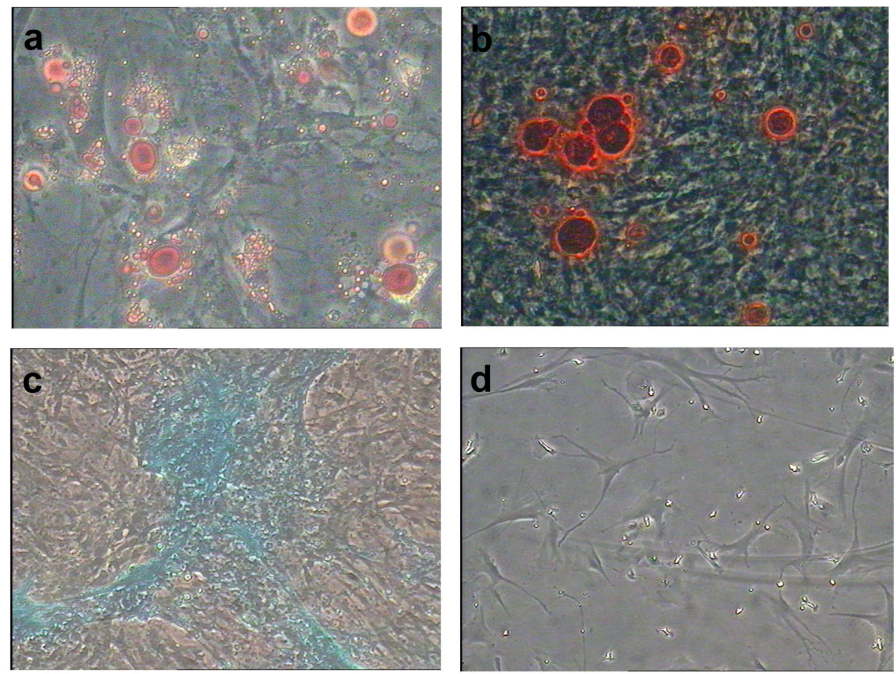

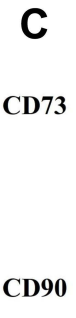 \\ C}
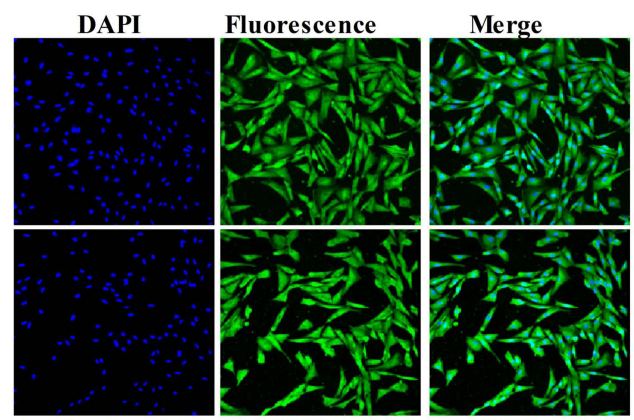

CD105
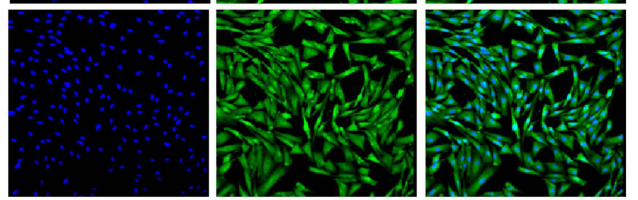

SOX2
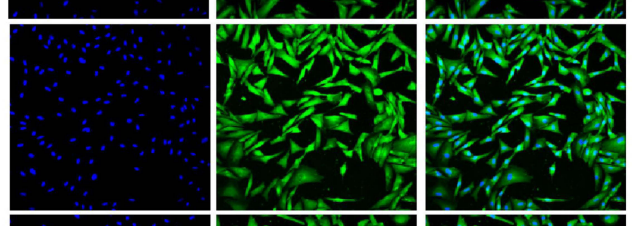

OCT4
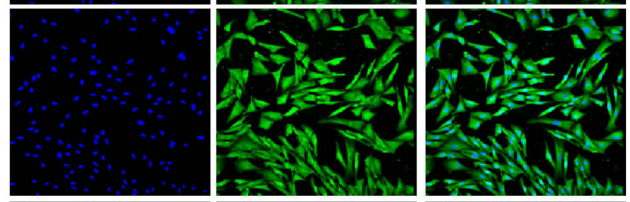

CD34
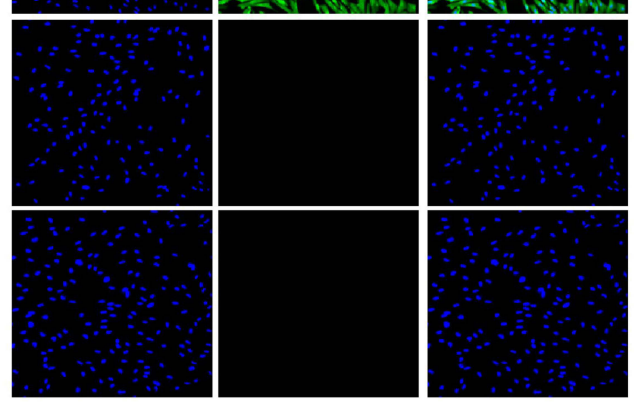

Figure I Biological characteristics of GMSCs.

Notes: (A) The cellular morphology of the GMSCs. The cellular morphology of the GMSCs was determined at different passages: I (A-a), P5 (A-b), PI5 (A-c), and P25 (A-d) (200x magnification). (B) Multidirectional differentiation potential of GMSCs. Under osteogenic induction conditions for 21 days, Alizarin Red staining was performed to show calcium deposition (B-a). Under adipocytic induction conditions for 14 days, Oil Red O staining was performed to evaluate the accumulation of lipids (B-b). Under chondrogenic induction conditions for 21 days, Alcian blue staining was performed to confirm chondrogenic differentiation (B-c) (200x magnification). Under neurogenic induction conditions for $2 \mathrm{I}$ days, the cellular morphology changed from a spindle-shaped morphology to a multipolar and stellate morphology, and the GMSCs grew many branches and formed many synapses on the cell surface, as indicated by the arrows (B-d) (400x magnification). (C) The results of immunofluorescent staining. GMSCs surface antigens were detected by immunofluorescence staining (200x magnification).

0.01), CXCL-1 $(P<0.01)$ and TNF- $\alpha(P<0.01)$ were markedly reduced in LPS + MSC group in comparison to LPS group.

\section{Oxidative Stress Parameters in Lung Tissue}

The expressions of SOD (Figure 2F), MDA (Figure 2G), GSH (Figure 2H), and MPO (Figure 2I) in lung tissue were detected. LPS administration prominently increased the expression of MDA $(P<0.05)$ and MPO $(P<0.05)$ and prominently decreased the expression of SOD $(P<$
0.05) and GSH $(P<0.05)$ compared to Sham. Significant decreases in the MDA $(P<0.05)$ and MPO $(P<0.05)$ levels and significant increases in $\operatorname{SOD}(P<0.05)$ and GSH $(P<0.05)$ levels were detected after GMSCs administration in comparison to LPS group.

\section{Morphological Assessment}

Compared with the lung tissues of normal mice (Figure 3A, D, and G), the degrees of lung injury, inflammatory infiltration, and interstitial edema increased after LPS administration (Figure 3B, E, and H). GMSCs 

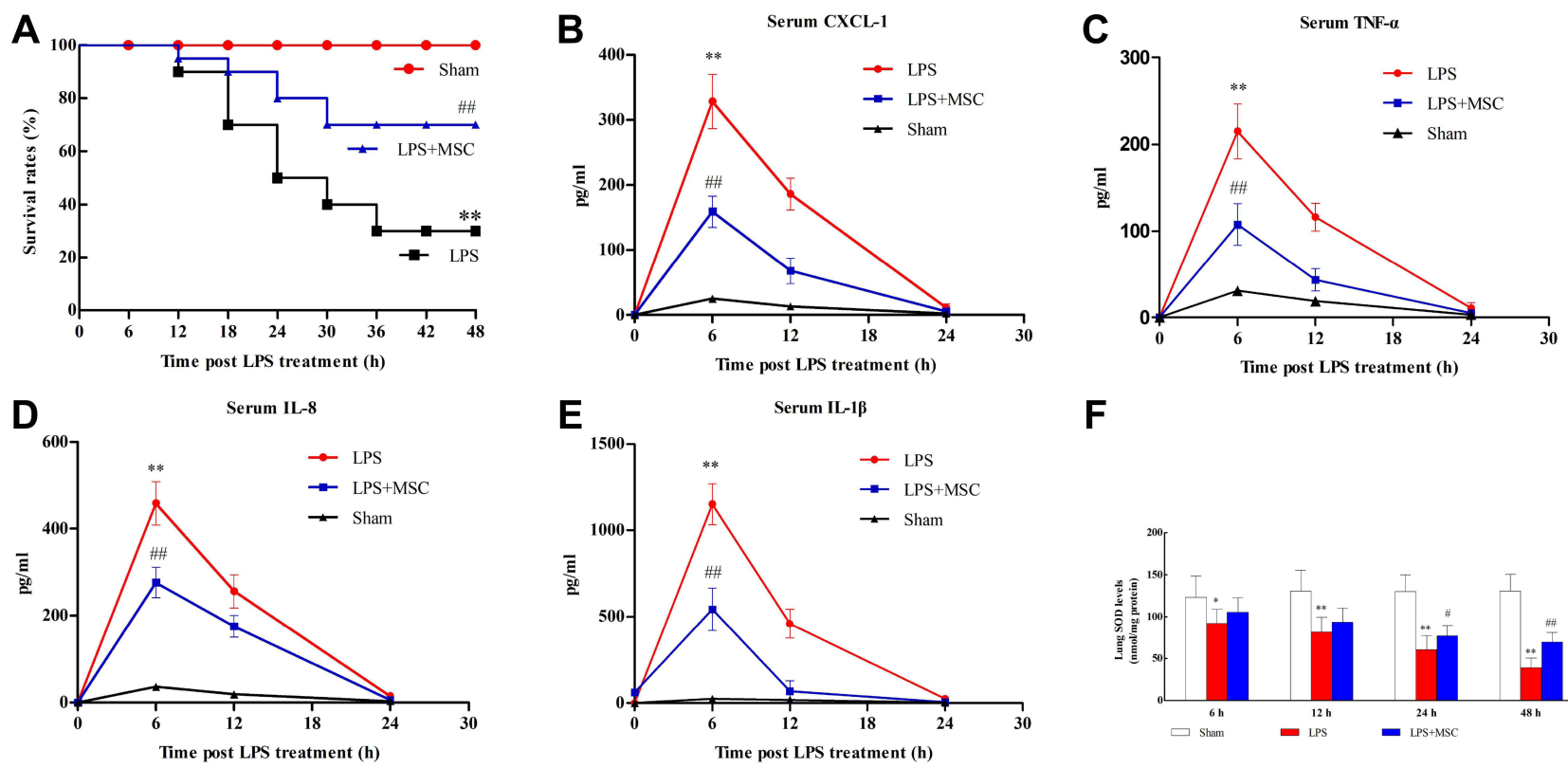

$\mathbf{F}$
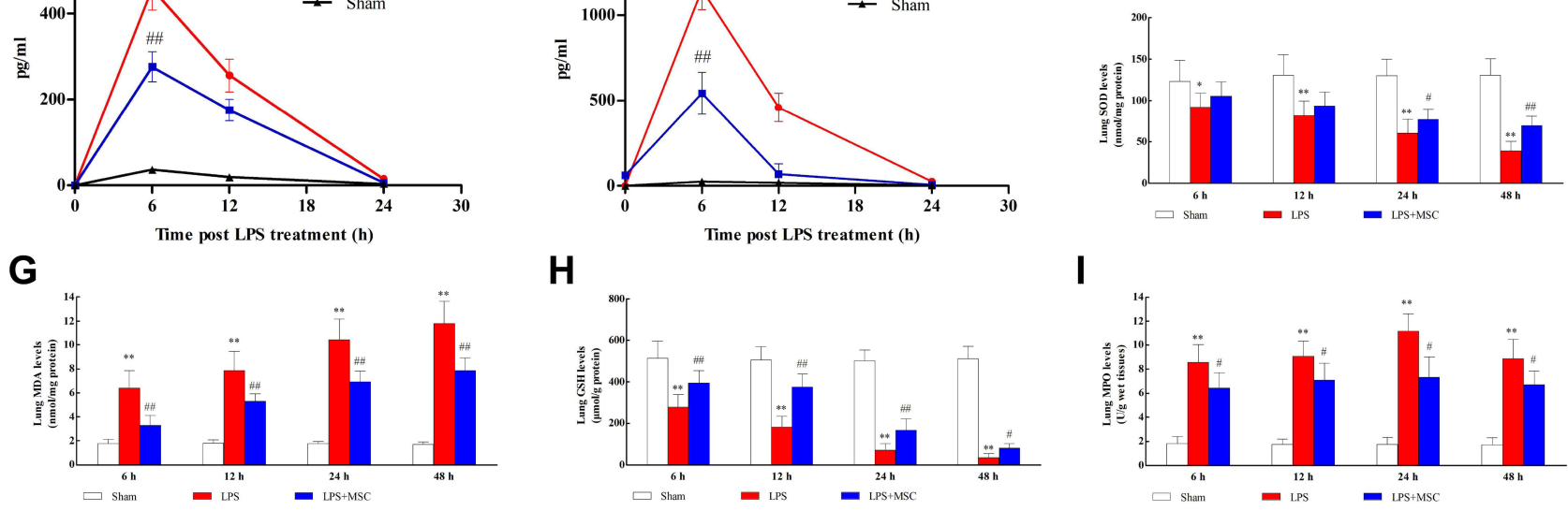

I

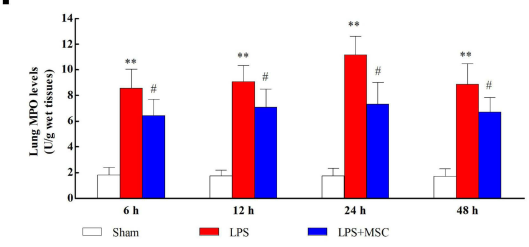

Figure 2 Assessment of survival rates, systemic inflammation, and oxidative stress injury.

Notes: (A) Assessment of survival rates. The serum levels of CXCL-I (B), TNF- $\alpha(\mathbf{C})$, IL-8 (D), and IL-I $\beta$ (E) were detected. The levels of SOD (F), MDA (G), GSH (H), and MPO (I) in lung tissue, as indicators of oxidative stress injury, were detected. ${ }^{*} P<0.05$ compared with the Sham group. ${ }^{\#} P<0.05$ compared with the LPS group. ${ }^{* * P}<$ 0.01 compared with the Sham group. ${ }^{\#} P<0.01$ compared with the LPS group. Values are expressed as the means \pm SD.

treatment significantly improved the degrees of lung injury, inflammatory infiltration, and interstitial edema (Figure 3C, F, and I) compared to LPS. As Supplementary Material 3 showed, GMSCs administration significantly decreased the lung injury score at each point in mice with sepsis $(P<0.05)$.

\section{Pulmonary Permeability}

LPS injection prominently upregulated the W/D $(P<0.05$; Figure 3J) and the concentration of total protein in BALF $(P<0.05$; Figure $3 \mathrm{~K})$ compared to Sham. GMSCs treatment exerted a prominent preventative effect on the increases in total protein concentration $(P<0.05)$ and the $\mathrm{W} / \mathrm{D}$ ratio $(P<0.05)$, demonstrating that GMSCs administration reduced the degree of pulmonary edema.

\section{Pulmonary Inflammation}

As shown in Table 1, LPS administration prominently upregulated the neutrophil content $(P<0.01)$ and the macrophage content $(P<0.01)$ in BALF. BM-MSCs treatment prominently prevented the increase in neutrophil content $(P<0.01)$ and macrophage content $(P<0.01)$ compared to LPS. LPS or BM-MSCs had no effect on the number of lymphocytes and eosinophils $(P>0.05)$.

As shown in Table 2, LPS injection prominently upregulated the BALF levels of neutrophil chemoattractants CXCL-1 $(P<0.01)$, IL-8 $(P<0.01)$, and TNF- $\alpha(P<$ $0.01)$, and proinflammatory factor IL-1 $\beta(P<0.01)$ and antiinflammatory factor IL-10 $(P<0.01)$; while LPS injection prominently downregulated anti- inflammatory factor IL$1 \mathrm{RN}(P<0.01)$. GMSCs administration prominently upregulated the BALF levels of IL-1RN $(P<0.01)$ and IL-10 $(P<0.01)$ but prominently downregulated the BALF levels of CXCL-1 $(P<0.01)$, IL-8 $(P<0.05)$, TNF- $\alpha(P<0.05)$, and IL-1 $\beta(P<0.05)$ at 6 hours after sepsis induction.

As shown in Table 2, LPS injection markedly upregulated BALF levels of CXCL-1 $(P<0.01)$, IL-6 $(P<0.01)$, IL-8 $(P<0.01)$, IL-10 $(P<0.01)$, and TNF- $\alpha(P<0.01)$ and markedly downregulated BALF levels of IL-1RN $(P<0.01)$. 




Figure 3 GMSCs administration reduces lung injury and lung permeability.

Notes: (A-I) Histologic assessment of lung tissues. The wet/dry ratios $(\mathbf{J})$ and the concentration of total protein in BALF (K) at each point were determined. ((A-I), I00x magnification). ${ }^{*} P<0.05$ compared with the Sham group. ${ }^{\#} P<0.05$ compared with the LPS group. ${ }^{* *} P<0.01$ compared with the Sham group. ${ }^{\# \#} P<0.01$ compared with the LPS group. Values are expressed as the means \pm SD.

BM-MSCs administration increased IL-10 $(P<0.01)$ and IL$1 \mathrm{RN}(P<0.05)$ release and impaired CXCL-1 $(P<0.05)$, IL$6(P<0.05)$, IL-8 $(P<0.05)$, and TNF- $\alpha(P<0.05)$ release in BALF in comparison to LPS group.

Table I Cell Counts in the BALF (Cells $/ \mathrm{mL})$ at 6 Hours PostSepsis

\begin{tabular}{|l|c|c|c|}
\hline Group & Sham & LPS & LPS + MSC \\
\hline Total cells & $1.35 \pm 1.21$ & $11.28 \pm 2.14^{* *}$ & $8.58 \pm 0.94^{\#}$ \\
Neutrophils & $0.04 \pm 0.03$ & $7.35 \pm 1.23^{* *}$ & $5.84 \pm 2.38^{\#}$ \\
Macrophages & $1.01 \pm 0.48$ & $3.12 \pm 0.67^{* *}$ & $2.56 \pm 0.54^{\#}$ \\
Lymphocytes & $0.04 \pm 0.07$ & $0.15 \pm 0.14$ & $0.15 \pm 0.16$ \\
Eosinophils & $0.02 \pm 0.04$ & $0.01 \pm 0.03$ & $0.04 \pm 0.05$ \\
\hline
\end{tabular}

Notes: Compared with the sham group, LPS administration increased the numbers of total cells, neutrophils, and macrophages. Compared with the LPS group, GMSCs administration decreased the numbers of total cells, neutrophils, and macrophages. ${ }^{\#} P<0.05$ compared with the LPS group, ${ }^{* *} P<0.01$ compared with the sham group, $\#^{\#} P<0.01$ compared with the LPS group. Values are expressed as the means \pm SD.

\section{Morphological Analysis of Aortic Injury}

In the sham group, the aorta was uniformly stained and arranged regularly, the endothelium was smooth and organized, and the elastic fibers had a regular, wavy shape (Figure 4A and D). After LPS administration, the endothelium was not smooth or regular, the elastic fibers of the media became sparse, and the elastic fibers lost their regular, wavy shape; furthermore, LPS administration significantly increased the aortic media thickness and the number of neutrophils and significantly decreased the area of the elastic fibers (Figure 4B and E). Compared with the LPS group, after GMSCs administration, mice in the LPS + MSC group showed decreased aortic media thickness and increased elastic fiber area; furthermore, in the LPS + MSC group, endothelial elastic fiber shape and elastic fiber distribution were improved, but these fibers had not completely returned to their normal form (Figure 4C and F). 
Table 2 Inflammatory Cytokine Levels in the BALF $(\mathrm{Pg} / \mathrm{mL})$ at 6 Hours Post-Sepsis

\begin{tabular}{|l|c|c|c|}
\hline Group & Sham & LPS & LPS + MSC \\
\hline CXCL-I & $16.2 \pm 1.86$ & $648.9 \pm 109.53^{* *}$ & $265.86 \pm 58 . I^{\# \#}$ \\
IL-I $\beta$ & $19.24 \pm 3.81$ & $706.9 \pm 161.5^{* *}$ & $563.1 \pm 116.9^{\#}$ \\
IL-8 & $13.9 \pm 5.1$ & $489.6 \pm 95.46^{* *}$ & $404.9 \pm 109.3^{\#}$ \\
IFN- $\gamma$ & $11.33 \pm 2.93$ & $152.3 \pm 18.9^{* *}$ & $128.6 \pm 23.9^{\#}$ \\
TNF- $\alpha$ & $19.32 \pm 3.38$ & $236.14 \pm 15.15^{* *}$ & $189.28 \pm 10.69^{\#}$ \\
IL-IRN & $50.37 \pm 9.48$ & $23.68 \pm 4.29^{* *}$ & $68.34 \pm 21.98^{\# \#}$ \\
IL-I0 & $16.9 \pm 3.7$ & $513.2 \pm 95.85^{* *}$ & $804.9 \pm 216.7^{\# \#}$ \\
\hline
\end{tabular}

Notes: Upregulation of the BALF levels of CXCL-I, IL-I I , IL-8, IL-I0, IFN- $\gamma$, and TNF- $\alpha$ and downregulation of the BALF levels of IL-IRN were detected after LPS injection, while GMSCs administration decreased the BALF levels of the proinflammatory factors CXCL-I, IL-I $\beta$, IL-8, IFN- $\gamma$, and TNF- $\alpha$ but increased the BALF levels of the anti-inflammatory factors IL-IRN and IL-I0 in mice with sepsis. ${ }^{\#} P<$ 0.05 compared with the LPS group, $* * P<0.01$ compared with the sham group, ${ }^{\#} P<0.01$ compared with the LPS group. Values are expressed as the means \pm SD.

Abbreviations: CXCL, C-X-C motif chemokine ligand; IL, interleukin; TNF, tumor necrosis factor; IFN, interferon.

As Table 3 shows, LPS administration increased the medial thickness of the aorta $(P<0.01)$ but decreased the median membrane elastic fiber area ratio $(P<0.01)$ in comparison to sham group. GMSCs administration decreased the medial thickness of the aorta $(P<0.05)$ and increased the median membrane elastic fiber area ratio $(P<0.01)$ in comparison to LPS group.

\section{GMSCs Attenuate LPS-Induced MODS GMSCs Attenuate Liver Injury}

The liver lobules of the mice in the sham group were intact and clear, the cells were neatly arranged, the intercellular substance was free of edema, the liver stripes were clear and regular, and there were no symptoms of injury (Figure 5A and D). The liver lobules of the mice in the LPS group were severely damaged, liver cells swelled, intercellular substances disappeared, and there was a large amount of neutrophil infiltration (Figure 5B and E). The liver lobules in the LPS + MSC group exhibited significantly less structural damage to liver tissues, with clearer liver lobules and a small amount of neutrophil infiltration (Figure 5C and F).

As Table 3 shows, LPS administration prominently upregulated serum levels of ALT $(P<0.01)$ and AST $(P<0.01)$ compared to sham. While GMSCs treatment prominently downregulated serum levels of ALT $(P<$ $0.05)$ and AST $(P<0.05)$ compared to LPS. As Supplementary Material 3 showed, GMSCs administration significantly decreased the liver injury score in mice with sepsis $(P<0.05)$.

\section{GMSCs Attenuate Kidney Injury}

The kidney tissues of the mice in the sham group (Figure 6A and D) were intact and clear, the cells were
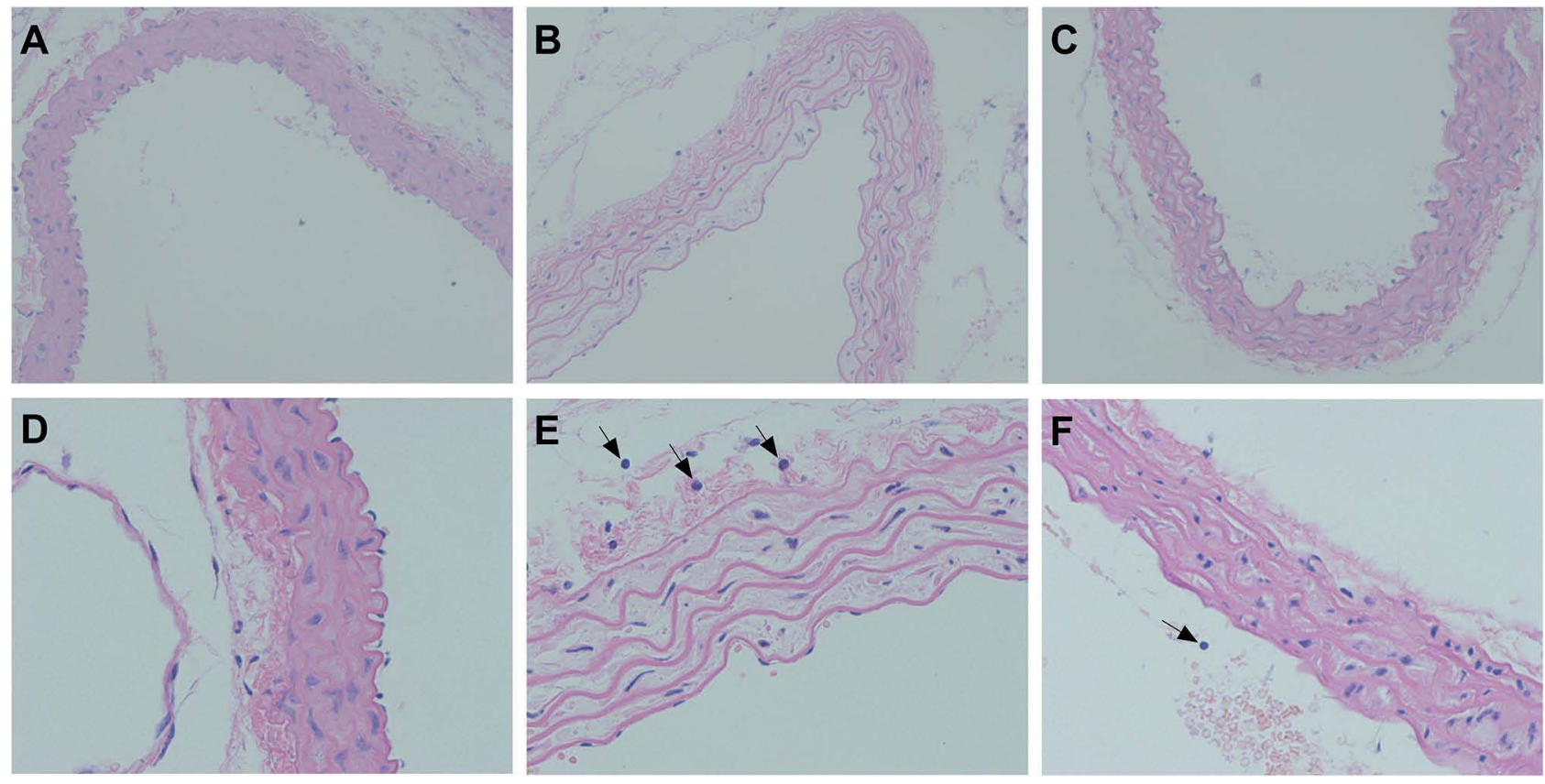

Figure 4 Histologic assessment of aortic tissues.

Notes: (A) Sham group. (B) LPS group. (C) LPS + MSC group. (D) Sham group. (E) LPS group. (F) LPS + MSC group. Histologic assessment revealed evidence of the degree of aortic injury. Black arrows indicate neutrophils. ((A-C), 100x magnification). ((D-F), 400x magnification). 
Table 3 Markers of Liver Disease and Kidney Injury at 48 Hours Post-Sepsis

\begin{tabular}{|c|c|c|c|}
\hline Group & Sham & LPS & LPS + MSC \\
\hline ALT (U/L) & $27.34 \pm 0.37$ & $48.55 \pm 9.86 * *$ & $37.21 \pm 7.46^{\#}$ \\
\hline AST (U/L) & $112.13 \pm 15.9$ & $160.94 \pm 49.6^{* *}$ & $130.45 \pm 26^{\#}$ \\
\hline Urea & $|8.3| \pm 3.24$ & $75.13 \pm 11.6 * *$ & $40.88 \pm 5.31^{\#}$ \\
\hline BUN (mmol/L) & $5.85 \pm 1.12$ & $8.28 \pm 1.6 I^{* *}$ & $7.0 \pm 1.55^{\#}$ \\
\hline $\operatorname{Scr}(m g / d l)$ & $0.11 \pm 0.02$ & $0.48 \pm 0.08^{* *}$ & $0.32 \pm 0.06^{\#}$ \\
\hline Medial thickness of aorta & $51.4 \pm 10.12$ & $65.46 \pm 11.8^{* *}$ & $57.79 \pm 11.8^{\#}$ \\
\hline Median membrane elastic fiber area ratio & $48.93 \pm 9.24$ & $27.45 \pm 3.23 * *$ & $41.27 \pm 5.89^{\# \#}$ \\
\hline
\end{tabular}

Notes: Upregulation of the levels of liver disease markers (ALT and AST), markers of kidney injury (urea, BUN, and Scr), medial thickness of the aorta, and median membrane elastic fiber area ratio were detected after LPS injection, while GMSCs administration prevented the upregulation of the levels of ALT, AST, urea, BUN, and Scr in serum. ${ }^{\#} P<0.05$ compared with the LPS group, ${ }^{* * P}<0.01$ compared with the sham group, ${ }^{\#} P<0.01$ compared with the LPS group. Values are expressed as the means \pm SD.

Abbreviations: ALT, alanine transaminase; AST, aspartate aminotransferase; BUN, blood urea nitrogen; Scr, serum creatinine.

neatly arranged, the intercellular substance was free of edema, cortical tubular epithelial cells were well shaped. The kidneys of septic mice were severely damaged, the cells swelled, and the intercellular substance disappeared. In addition, severe epithelial vacuolization and an atypical shape with almost no nuclei were observed in septic mice (Figure 6B and E). Compared with the LPS group, septic mice had significantly less kidney tissue structural damage, clearer nephrons and less vacuolization after GMSCs administration (Figure 6C and F), suggesting that GMSCs administration reduced LPS-induced kidney injury.
As Table 3 shows, serum levels of urea $(P<0.01)$, BUN $(P<0.01)$, and Scr $(P<0.01)$ in the LPS group were prominently upregulated compared to sham, while GMSCs treatment prominently downregulated urea $(P<0.05)$, BUN $(P<0.05)$, and Scr $(P<0.05)$ compared to LPS. As Supplementary Material 3 showed, GMSCs administration significantly decreased the acute tubular damage score in mice with sepsis $(P<0.05)$.

\section{GMSCs Attenuate Heart Injury}

In the sham group, the myocardial tissue was uniformly stained, the myocardial fibers were arranged regularly, and
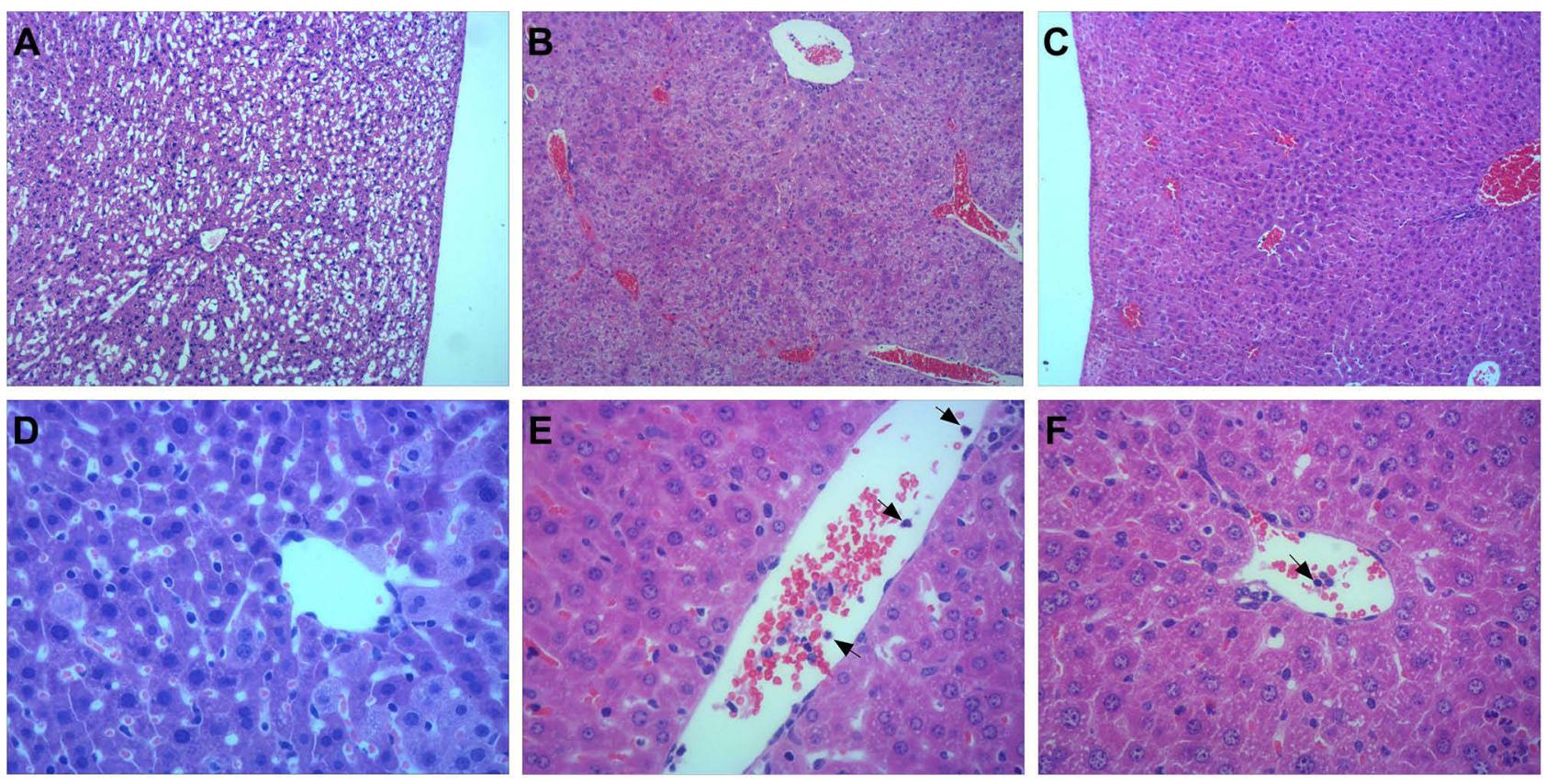

Figure 5 Histologic assessment of liver tissues.

Notes: (A) Sham group. (B) LPS group. (C) LPS + MSC group. (D) Sham group. (E) LPS group. (F) LPS + MSC group. Histologic assessment revealed evidence of the degree of liver injury. Black arrows indicate neutrophils. ((A-C), 100x magnification). ((D-F), 400x magnification). 

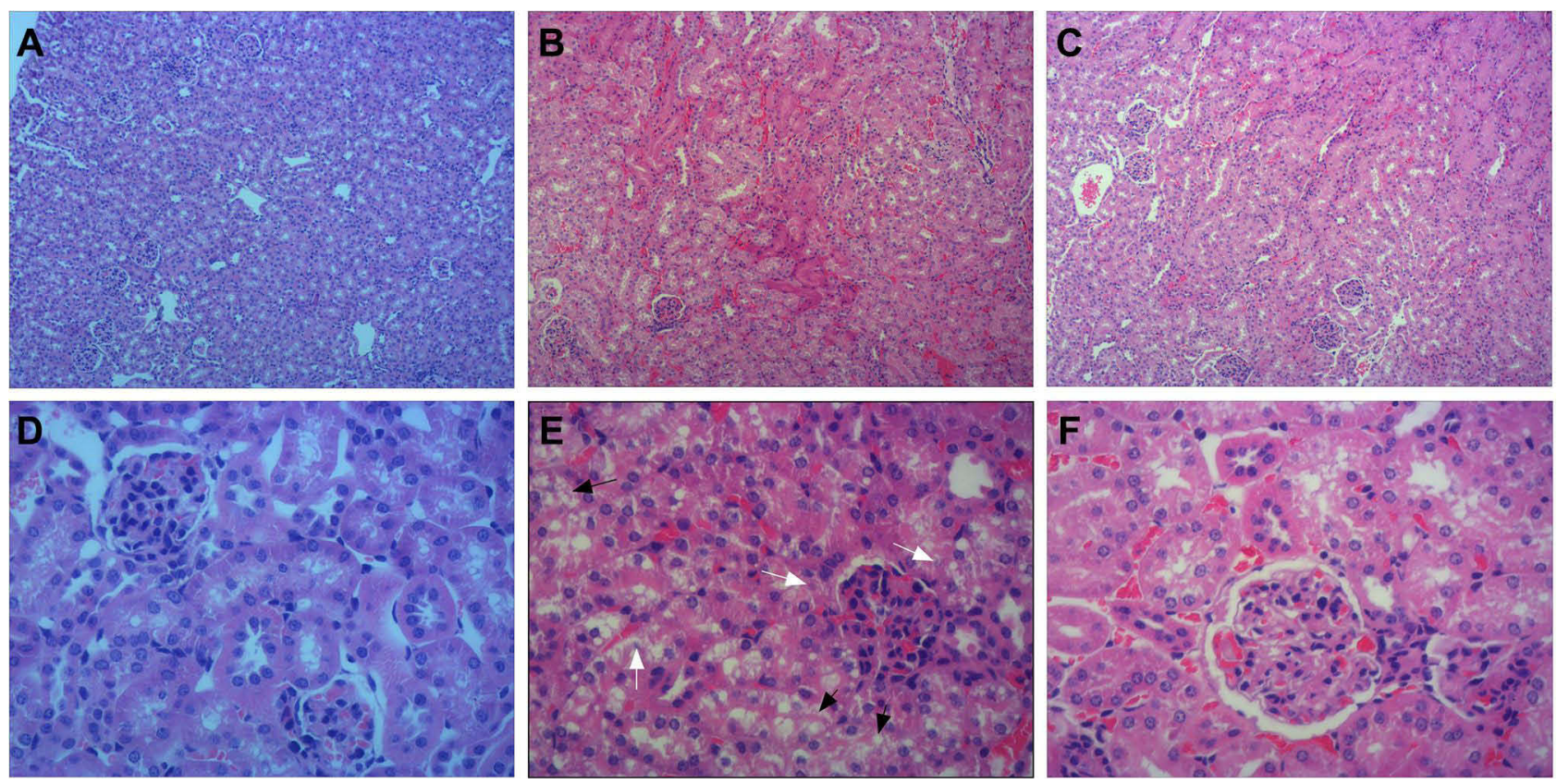

Figure 6 Histologic assessment of kidney tissues.

Notes: (A) Sham group. (B) LPS group. (C) LPS + MSC group. (D) Sham group. (E) LPS group. (F) LPS + MSC group. Histologic assessment revealed evidence of the degree of kidney injury. Septic mice had severe epithelial vacuolization (black arrows) and an atypical shape with almost no nuclei (white arrows). ((A-C), I00x magnification). ((DF), 400x magnification).

the interstitial spaces were regular (Figure 7A). In the LPS group, myocardial tissues were disordered, myocardial degeneration occurred, dissolution was observed, and a large number of inflammatory cells infiltrated the muscle space (Figure 7B).

After GMSCs treatment, the inflammatory cells were less infiltrated, and the myocardial fiber tissue structure was restored in mice with sepsis. But the muscle fibers were still partially dissolved. The distribution of muscle fibers was improved, but it did not completely return to the normal form (Figure 7C). As Supplementary Material 3 showed, GMSCs administration significantly decreased the myocardial injury score in mice with sepsis $(P<0.05)$.

\section{GMSCs Regulate the Warburg Effect During Sepsis}

LPS injection markedly increased the levels of lactate $(P<$ 0.05 ; Figure $8 \mathrm{~A})$ and HMGB1 in serum $(P<0.05$; Figure $8 \mathrm{C})$ but markedly decreased the blood glucose levels $(P<0.05$; Figure $8 \mathrm{~B}$ ) compared to sham. GMSCs treatment markedly increased blood glucose levels $(P<0.05)$ but decreased the serum levels of lactate $(P<0.05)$ and HMGB1 $(P<0.05)$ compared to LPS. LPS administration prominently upregulated GLUT1 $(P<0.01$; Figure 8D) and HMGB1 $(P<0.01$; Figure $8 \mathrm{E}$ ) mRNA expression levels in lung tissue, while GMSCs administration decreased GLUT1 $(P<0.01)$ and HMGB1 $(P<0.01)$ mRNA expression levels in lung tissue.
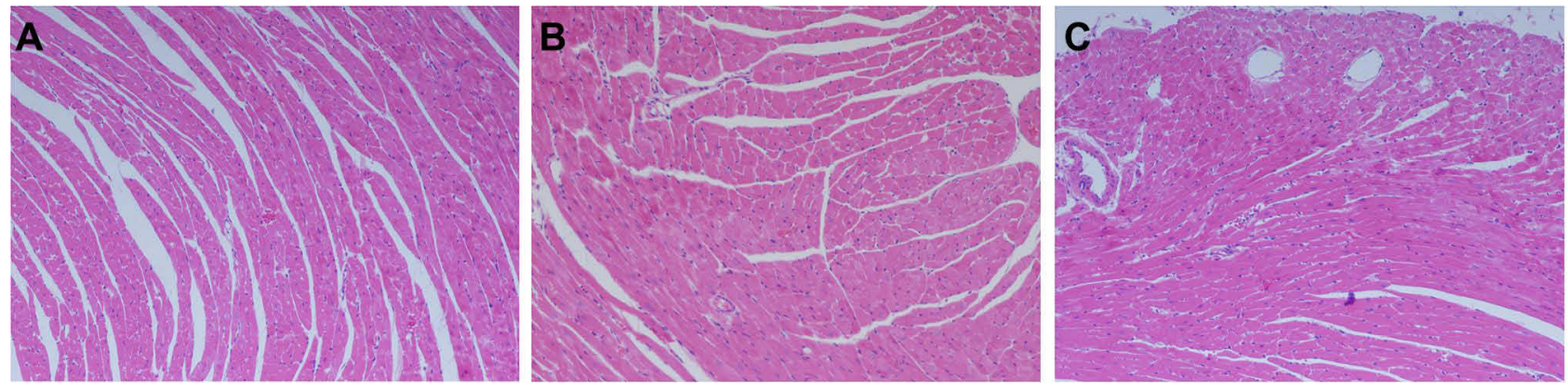

Figure 7 Histologic assessment of heart tissues.

Notes: (A) Sham group. (B) LPS group. (C) LPS + MSC group. Histologic assessment revealed evidence of the degree of heart injury. ((A-C), I00x magnification). 

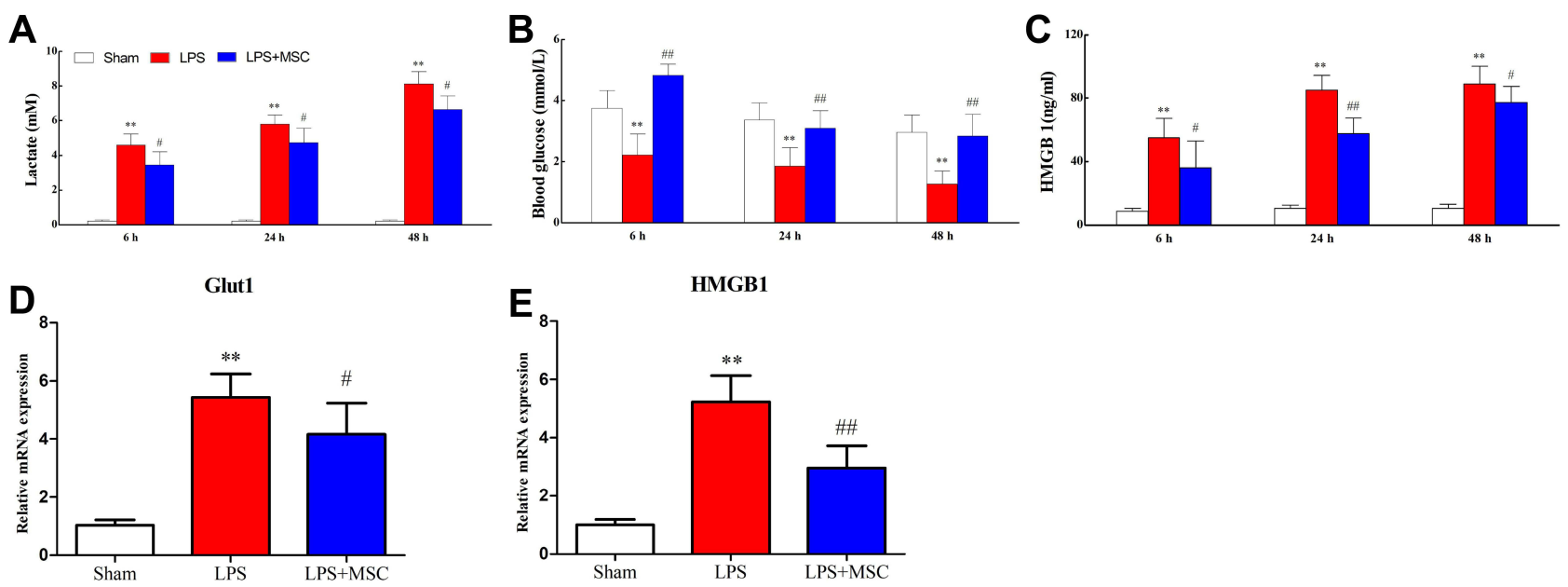

Figure 8 GMSCs impaired LPS-induced lactate and HMGBI release during sepsis.

Notes: (A) Detection of the levels of lactate in serum. (B) Detection of the levels of blood glucose. (C) Detection of the levels of HMGBI in serum. (D) Detection of the mRNA expression levels of Glutl in lung tissue. (E) Detection of the mRNA expression levels of HMGBI in lung tissue. ${ }^{\#} P<0.05$ compared with the LPS group. **P $<0.01$ compared with the Sham group. ${ }^{\#} P<0.01$ compared with the LPS group. Values are expressed as the means \pm SD.

Abbreviation: HMGBI, high-mobility group box $\mathrm{I}$.

\section{GMSCs Exert Immune Effects and Suppress the Excessive Inflammatory Response Induced by LPS}

Compared with the sham group, LPS injection prominently upregulated CXCL-1 $(P<0.01$; Figure 9A), IL-8 $(P<0.01$; Figure 9B), TNF- $\alpha(P<0.01$; Figure 9C), IFN- $\gamma$ $(P<0.01$; Figure 9D), TLR4 $(P<0.01$; Figure 9E), and Myd88 $(P<0.01$; Figure 9F) mRNA expression levels but prominently downregulated anti-inflammatory factors IL1RN $(P<0.01$; Figure 9G) and IL-10 $(P<0.01$, Figure $9 \mathrm{H}$ ) mRNA expression levels in lung tissue.
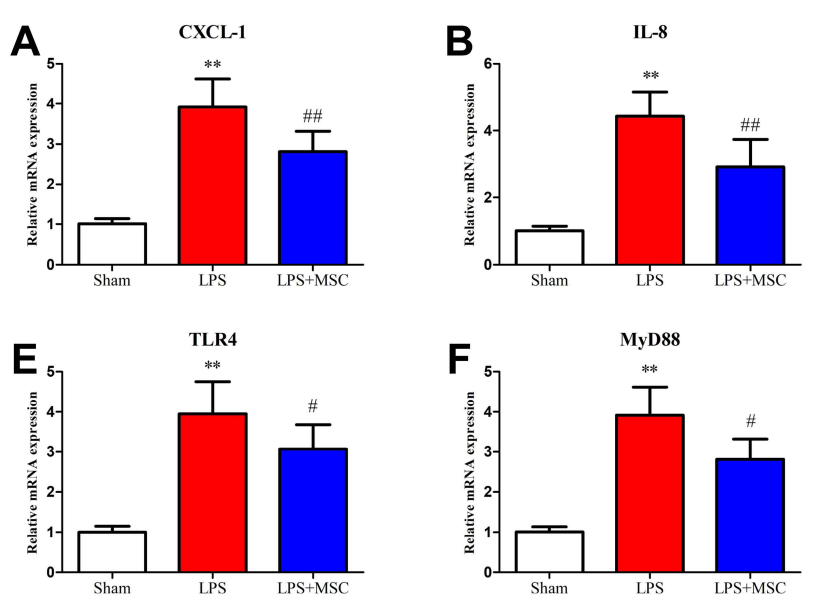

Compared with the LPS group, GMSCs treatment prominently downregulated CXCL-1 $(P<0.01)$, IL-8 $(P<$ $0.01)$, TNF- $\alpha(P<0.01)$, IFN- $\gamma(P<0.05)$, TLR4 $(P<$ $0.01)$ and $\operatorname{Myd} 88(P<0.01)$ mRNA expression levels of but prominently upregulated IL-1RN $(P<0.01)$ and IL-10 $(P<0.01)$ mRNA expression levels in lung tissue.

\section{Detection of CM-Dil-Labeled GMSCs}

To identify the homing of GMSCs, GMSCs were treated with CM-Dil (C7000, Life Technologies, Eugene, Oregon, USA). Supplementary Material 4 listed the murine PCR primer sequence information.
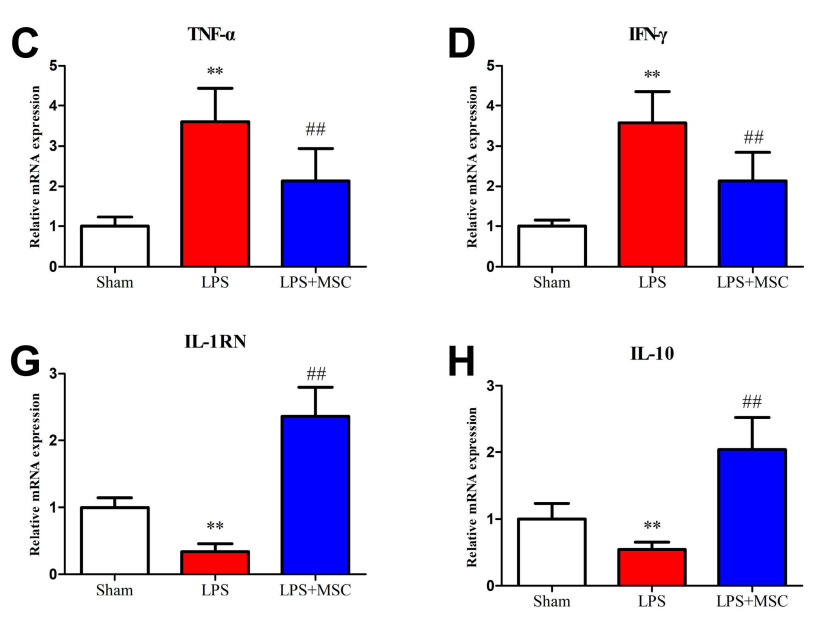

Figure 9 Detection of the mRNA expression levels in lung tissue.

Notes: The mRNA expression levels of CXCL-I (A), IL-8 (B), TNF- $\alpha(\mathbf{C})$, IFN- $\gamma(\mathbf{D})$, TLR4 (E), Myd88 (F), IL-IRN (G), and IL-I0 (H) in lung tissue were detected. ${ }^{P} P<$ 0.05 compared with the LPS group. ${ }^{* * P}<0.01$ compared with the Sham group. ${ }^{\#} P<0.01$ compared with the LPS group. Values are expressed as the means \pm SD. Abbreviations: CXCL, C-X-C motif chemokine ligand; IL, interleukin; TNF, tumor necrosis factor; IFN, interferon. 


\section{Discussion}

GMSCs, which were first separated and characterized in 2009, are a wealth of MSCs sources and can be harvested at low cost. ${ }^{42}$ Using gingival tissues separated from mice, we obtained a novel population of cells whose biological characteristics included several unique characteristics of MSCs, including a spindle-like morphology, multilineage differentiation potential, and a similar cell surface marker profile. These characteristics were similar to those of bone marrow- and adipose-derived MSCs. Gingival were unique masticatory keratinized mucosal tissues and owned rapid wound healing property. ${ }^{43,44}$ GMSCs had neural crest origin; hence, GMSCs can differentiate into neural cell lineages. ${ }^{45,46}$ Our data demonstrate that GMSCs can be induced to successfully differentiate into cells of mesodermal origin, such as osteoblasts, lipoblasts, and chondrocytes, and cells of ectodermal origin, such as neuroblasts. Hence, GMSCs were used to treat skin diseases, peripheral nerve injuries, and oral and maxillofacial disorders. These properties offer a scientific basis for GMSCs and make them promising alternatives for cellular transplantation therapy.

Previous studies demonstrated that the Warburg effect involved innate and adaptive immunity. ${ }^{25,26}$ There was convincing evidence that activated immune cells, including macrophages, neutrophils, and $\mathrm{T}$ cells, switched from phosphorylation to aerobic glycolysis during sepsis. ${ }^{25}$ Increased aerobic glycolysis consumes a large amount of glucose and produces a large amount of lactate. ${ }^{25}$ Previous studies found that increased serum lactate levels were a biological marker mortality and multiple system organ failure during sepsis and lactate clearance was a novel way of treatment for sepsis. ${ }^{26}$ HMGB1 is a promising therapeutic target for sepsis treatment. ${ }^{27,28}$ Previous studies found that GLUT1 stimulated macrophages to release HMGB1. ${ }^{26}$ Strikingly, our data demonstrated that LPS injection prominently upregulated the serum levels of lactate and HMGB1 and GLUT1 and HMGB1 mRNA expression levels in the lung. GMSCs administration decreased the levels of lactate and HMGB1 in serum and the mRNA expression levels of GLUT1 and HMGB1 in the lung. Based on these results, GMSCs improved MODS and systemic inflammatory reactions by impairing LPSinduced lactate, GLUT1, and HMGB1 release during sepsis. Our research identified a new mechanism through which GMSCs protect against sepsis.
Some studies found that LPS administration resulted in hyperglycemia. ${ }^{47,48}$ However, other studies found that LPS administration resulted in hypoglycemia. ${ }^{49-51}$ Our data revealed that LPS administration led to hypoglycemia partly because increased aerobic glycolysis consumed a large amount of blood glucose during sepsis. Hypoglycemia is a common complication of sepsis. Our data found that LPS administration prominently decreased blood glucose level, which was consistent with previous experimental results. ${ }^{49,50}$ If sepsis-induced hypoglycemia is not treated promptly, patients can deteriorate further and develop a coma, which is easily confused with a coma caused by infection, leading to delayed diagnosis and treatment or even death. Hence, septicemia patients should be monitored, and blood glucose should be supplemented in a timely manner. Strikingly, we demonstrated that GMSCs prevented a decrease in blood glucose levels. These results demonstrate that GMSCs can be used as a preventive tool for sepsis-induced hypoglycemia.

Our data demonstrated that LPS injection led to an excessive inflammatory response, pulmonary edema, and the infiltration of inflammatory cells, the three main features of ALI. ${ }^{7}$ The GMSCs regimen improved the degree of pulmonary edema, the degree of neutrophil infiltration, and ALI. Based on these results, GMSCs administration is a potential treatment for LPS-induced ALI.

Sepsis is an excessive and uncontrolled inflammatory response involving inflammatory mediators and effector cells, such as neutrophils. ${ }^{21}$ The deleterious accumulation of neutrophils led to host tissue injury and organ damage during sepsis. ${ }^{23}$ We demonstrated that LPS injection resulted in deleterious accumulation of neutrophils in organ, and our experimental results are consistent with previous conclusions. ${ }^{6}$ Strikingly, we found that GMSCs prominently reduced neutrophil content in organ. Our data demonstrated that GMSCs improved sepsis-induced lung injury in part by decreasing the neutrophil content. CXCL1, IL-8, and TNF- $\alpha$ are well known to be chemotactic for neutrophils. Our data showed that GMSCs treatment prominently decreased BALF levels of CXCL-1, IL-8, and TNF- $\alpha$ and CXCL-1, IL- 8 , and TNF- $\alpha$ mRNA expression levels in the lung. These results demonstrated that GMSCs inhibited neutrophil infiltration in part by suppressing CXCL-1, IL-8, and TNF- $\alpha$ expression. In the present study, the abnormal accumulation of neutrophils in organ was observed in septic mice. GMSCs administration prevented this abnormal accumulation of neutrophils in organ. Based on these results, GMSCs administration protected 
against MODS partly because GMSCs reduced the abnormal accumulation of neutrophils by suppressing CXCL-1, IL-8, and TNF- $\alpha$.

Previous studies have demonstrated that TLR4 can stimulate human artery endothelial cells to release proinflammatory factors, including TNF- $\alpha$ and IL-1 $\beta .^{52,53}$ Our data demonstrated that LPS administration prominently upregulated TLR4, Myd88, IL-1 $\beta$, and TNF- $\alpha$ mRNA expression, while GMSCs administration prominently upregulated TLR4, Myd88, and IL-1 $\beta$, and TNF- $\alpha$ mRNA expression. Besides, GMSCs administration prevented an increase in the levels of TNF- $\alpha$ and IL-1 $\beta$ in BALF and serum during sepsis.

Vascular injury occurs before the onset of inflammatory infiltration and organ injury. ${ }^{15}$ Previous studies overlooked the effects of sepsis and GMSCs on the aorta. The inhibiting effect of GMSCs on TLR4/MyD88 signaling during sepsis was demonstrated. Our data demonstrated that LPS injection increased the aortic medial thickness and reduced the area ratio of elastic fibers, which was reversed by GMSCs administration. Based on these results, GMSCs are a potential treatment for LPSinduced aortic injury.

Our data demonstrated that LPS injection led to an excessive inflammatory response, pulmonary edema, and the infiltration of inflammatory cells, the three main features of ALI. ${ }^{7}$ The GMSCs regimen improved the degree of pulmonary edema, the degree of neutrophil infiltration, and ALI. Based on these results, GMSCs administration is a potential treatment for LPS-induced ALI.

LPS administration resulted in oxidative stress injury. ${ }^{35}$ Our data showed found that GMSCs had an antioxidant effect. We demonstrated that GMSCs prominently downregulated MDA and MPO expression but prominently upregulated SOD and GSH expression in mice with sepsis. Hence, GMSCs treatment modulated the oxidative/antioxidative balance in lung tissue. Thus, GMSCs treatment was a preventive tool for sepsis-associated lung injury owing to its antioxidant capacity.

MSCs reduced LPS-induced systemic inflammation. ${ }^{36}$ Our data demonstrated that treatment with GMSCs prominently upregulated serum levels of IL-10 and IL-1RN but prominently downregulated serum levels of CXCL-1, IL$1 \beta$, and IL- 8 during sepsis. Hence, GMSCs administration reduced LPS-induced systemic inflammation.

The LPS model is most suitable when analyzing the effects of novel treatments for acute inflammation. ${ }^{56-59}$ Hence, we chose LPS instead of CLP to establish a sepsis model. The inherent immunosuppressive properties and low immunogenicity of MSCs suggest their therapeutic potential in transplantation. Some studies have suggested that MSCs have low immunogenicity, while other studies have shown that MSCs are not immunogenic. We hypothesized that MSCs exhibited low immunogenicity. To avoid the experiment being affected by uncontrollable factors, including immunological rejection, ALL mice were intraperitoneally injected with the anti-rejection drug cyclosporine for one week before the experiment. Intraperitoneal injection of cyclosporine was not a necessary measure for stem cell therapy. Our data for mice treated with LPS showed that the serum levels of four proinflammatory cytokines, IL-1 $\beta$, TNF- $\alpha$, IL-8, and IL-6, were increased within $6 \mathrm{~h}$, decreased at $12 \mathrm{~h}$, and decreased again at $24 \mathrm{~h}$. GMSCs prevented the upregulation of these four proinflammatory cytokines in the serum. Generally, stem cells remained in the body for 48 hours. After 48 hours, the stem cells will be excreted from the body. Hence, we only evaluated the mortality rate of the mice within $48 \mathrm{~h}$.

In conclusion, our results showed that GMSCs improved LPS-induced death, hypoglycemia, and organ injury, including lung, liver, kidney, heart, and aortic injury. GMSCs exerted immune effects, reduced neutrophil inflammation, suppressed oxidative stress injury, and impaired LPS-induced lactate and HMGB1 release.

\section{Funding}

This study was supported by the Agricultural Science and Technology Innovation Program of China (ASTIP-IAS01), the National Germplasm Center of Domestic Animal Resources, National Natural Science Foundation of China (NSFC) (Grant No. 71874017, 81472992), and the BNU Interdisciplinary Research Foundation for the First-Year Doctoral Candidates (Grant No. BNUXKJC1814).

\section{Disclosure}

The authors report no conflicts of interest in this work. The manuscript has been approved by all authors for publication.

\section{References}

1. Guo F, Shen H. Glycosylated hemoglobin as a predictor of sepsis and all-cause mortality in trauma patients. Infect Drug Resist. 2021;14:2517-2526. doi:10.2147/IDR.S307868

2. Li D, Weng Y, Wang G, et al. Anti-septic potential of 7- $\alpha$-obacunyl acetate isolated from the toona sinensis on cecal ligation/puncture mice via suppression of JAK-STAT/NF- $\mathrm{B}$ signal pathway. Infect Drug Resist. 2021;14:1813-1821. doi:10.2147/IDR.S302853

3. Kusadasi N, Groeneveld AB. A perspective on mesenchymal stromal cell transplantation in the treatment of sepsis. Shock. 2013;40:352-357. doi:10.1097/SHK.0000000000000039 
4. Matthay MA, Thompson BT, Read EJ, et al. Therapeutic potential of mesenchymal stem cells for severe acute lung injury. Chest. 2010;138:965-972.

5. Chamberlain G, Fox J, Ashton B, Middleton J. Concise review: mesenchymal stem cells: their phenotype, differentiation capacity, immunological features, and potential for homing. Stem Cells. 2007;25:2739-2749. doi:10.1634/stemcells.2007-0197

6. de Witte SFH, Luk F, Sierra Parraga JM, et al. Immunomodulation by therapeutic Mesenchymal Stromal Cells (MSC) is triggered through phagocytosis of MSC by monocytic cells. Stem Cells. 2018;36 (4):602-615. doi:10.1002/stem.2779

7. Gupta N, Su X, Popov B, et al. Intrapulmonary delivery of bone marrow-derived mesenchymal stem cells improves survival and attenuates endotoxin-induced acute lung injury in mice. J Immunol. 2007;179:1855-1863. doi:10.4049/jimmunol.179.3.1855

8. Rojas M, Xu J, Woods CR, et al. Bone marrow-derived mesenchymal stem cells in repair of the injured lung. Am J Respir Cell Mol Biol. 2005;33:145-152. doi:10.1165/rcmb.2004-03300C

9. Serikov VB, Mikhaylov VM, Krasnodembskay AD, et al. Bone marrow-derived cells participate in stromal remodeling of the lung following acute bacterial pneumonia in mice. Lung. 2008;186:179-190. doi:10.1007/s00408-008-9078-6

10. Gupta N, Su X, Popov B, et al. Intrapulmonary delivery of bone marrow-derived mesenchymal stem cells improves survival and attenuates endotoxin-induced acute lung injury in mice. $J$ Immunol. 2007;179:1855-1863. doi:10.4049/jimmunol.179.3.1855

11. Mei SH, McCarter SD, Deng Y, et al. Prevention of LPS-induced acute lung injury in mice by mesenchymal stem cells overexpressing angiopoietin 1. PLoS Med. 2007;4:e269. doi:10.1371/journal. pmed.0040269

12. Luo CJ, Zhang FJ, Zhang L, et al. Mesenchymal stem cells ameliorate sepsis-associated acute kidney injury in mice. Shock. 2014;41:123-129. doi:10.1097/SHK.0000000000000080

13. Weil BR, Herrmann JL, Abarbanell AM, et al. Intravenous infusion of mesenchymal stem cells is associated with improved myocardial function during endotoxemia. Shock. 2011;36:235-241. doi:10.1097/ SHK.0b013e318225f6ae

14. Mei SH, Haitsma JJ, Dos Santos CC, et al. Mesenchymal stem cells reduce inflammation while enhancing bacterial clearance and improving survival in sepsis. Am $J$ Respir Crit Care Med. 2010;182:1047-1057. doi:10.1164/rccm.201001-0010OC

15. Xu J, Woods CR, Mora AL, et al. Prevention of endotoxin-induced systemic response by bone marrow-derived mesenchymal stem cells in mice. Am J Physiol Lung Cell Mol Physiol. 2007;293:131-141. doi:10.1152/ajplung.00431.2006

16. Pati S, Khakoo AY, Zhao J, et al. Human mesenchymal stem cells inhibit vascular permeability by modulating vascular endothelial cadherin/beta-catenin signaling. Stem Cells Dev. 2011;20:89-101. doi:10.1089/scd.2010.0013

17. Xiao K, He W, Guan W, et al. Mesenchymal stem cells reverse EMT process through blocking the activation of NF- $\mathrm{KB}$ and Hedgehog pathways in LPS-induced acute lung injury. Cell Death Dis. 2020;11:863.

18. Fang X, Neyrinck AP, Matthay MA, et al. Allogeneic human mesenchymal stem cells restore epithelial protein permeability in cultured human alveolar type II cells by secretion of angiopoietin-1. J Biol Chem. 2010;285:26211-26222. doi:10.1074/jbc.M110.119917

19. Goolaerts A, Pellan-Randrianarison N, Larghero J, et al. Conditioned media from mesenchymal stromal cells restores sodium transport and preserves epithelial permeability in an in vitro model of acute alveolar injury. Am J Physiol Lung Cell Mol Physiol. 2014;306:975-985. doi:10.1152/ajplung.00242.2013

20. Weiss SL, Peters MJ, Alhazzani W, et al. Surviving sepsis campaign international guidelines for the management of septic shock and sepsis-associated organ dysfunction in children. Intensive Care Med. 2020;46:10-67. doi:10.1007/s00134-019-05878-6
21. Walter J, Ware LB, Matthay MA. Mesenchymal stem cells: mechanisms of potential therapeutic benefit in ARDS and sepsis. Lancet Respir Med. 2014;2(12):1016-1026. doi:10.1016/S2213-2600(14) 70217-6

22. Ahn SY, Maeng YS, Kim YR, et al. In vivo monitoring of dynamic interaction between neutrophil and human umbilical cord blood-derived mesenchymal stem cell in mouse liver during sepsis. Stem Cell Res Ther. 2020;11:44-47. doi:10.1186/s13287-020-1559-4

23. Shen XF, Cao K, Jiang JP, et al. Neutrophil dysregulation during sepsis: an overview and update. $J$ Cell Mol Med. 2017;21 (9):1687-1697. doi:10.1111/jcmm.13112

24. Jung YJ, Park YY, Huh JW, et al. The effect of human adipose-derived stem cells on lipopolysaccharide-induced acute respiratory distress syndrome in mice. Ann Transl Med. 2019;7 (22):674. doi:10.21037/atm.2019.10.48

25. Vander Heiden MG, Cantley LC, Thompson CB. Understanding the Warburg effect: the metabolic requirements of cell proliferation. Science. 2009;324(5930):1029-1033. doi:10.1126/science.1160809

26. Wen H, Ting JPY, O'Neill LAJ, et al. A role for the NLRP3 inflammasome in metabolic diseases-did Warburg miss inflammation? Nat Immunol. 2012;13:352-357. doi:10.1038/ni.2228

27. Yang L, Xie M, Yang M, et al. PKM2 regulates the Warburg effect and promotes HMGB1 release in sepsis. Nat Commun. 2014;5:4436. doi: $10.1038 /$ ncomms 5436

28. Bakker J, Coffernils M, Leon M, et al. Blood lactate levels are superior to oxygen-derived variables in predicting outcome in human septic shock. Chest. 1991;99:956-962. doi:10.1378/ chest.99.4.956

29. Régnier MA, Raux M, Le Manach Y, et al. Prognostic significance of blood lactate and lactate clearance in trauma patients. Anesthesiology. 2012;117:1276-1288. doi:10.1097/ALN.0b013e318273349d

30. Shapiro NI, Howell MD, Talmor D, et al. Serum lactate as a predictor of mortality in emergency department patients with infection. Ann Emerg Med. 2005;45:524-528. doi:10.1016/j.annemergmed.2004.12.006

31. Scaffidi P, Misteli T, Bianchi ME. Release of chromatin protein HMGB1 by necrotic cells triggers inflammation. Nature. 2002;418:191-195. doi:10.1038/nature00858

32. Wang H, Yang H, Czura CJ, et al. HMGB1 as a late mediator of lethal systemic inflammation. Am $J$ Respir Crit Care Med. 2001;164:1768-1773. doi:10.1164/ajrccm.164.10.2106117

33. Lotze MT, Tracey KJ. High-mobility group box 1 protein (HMGB1): nuclear weapon in the immune arsenal. Nat Rev Immunol. 2005;5 (4):331-342. doi:10.1038/nri1594

34. O'Neill LAJ, Hardie DG. Metabolism of inflammation limited by AMPK and pseudo-starvation. Nature. 2013;493(7432):346-355. doi:10.1038/nature 11862

35. Collins T, Read MA, Neish AS, et al. Transcriptional regulation of endothelial cell adhesion molecules: NF-kappa B and cytokine-inducible enhancers. FASEB J. 1995;9(10):899-909. doi:10.1096/fasebj.9.10.7542214

36. Walton KA, Hsieh X, Gharavi N, et al. Receptors involved in the oxidized 1-palmitoyl-2-arachidonoyl-sn-glycero-3-phosphorylcholine-mediated synthesis of interleukin- 8 . A role for Toll-like receptor 4 and a glycosylphosphatidylinositol-anchored protein. J Biol Chem. 2003;278(32):29661-29666. doi:10.1074/jbc.M300738200

37. Perrin-Cocon L, Aublin-Gex A, Sestito SE, et al. TLR4 antagonist FP7 inhibits LPS-induced cytokine production and glycolytic reprogramming in dendritic cells, and protects mice from lethal influenza infection. Sci Rep. 2017;7(1):40791. doi:10.1038/srep40791

38. Raetz CR, Whitfield C. Lipopolysaccharide endotoxins. Annu Rev Biochem. 2002;71:635-700. doi:10.1146/annurev.biochem.71.1 10601.135414

39. Eggenhofer E, Renner P, Soeder Y, et al. Features of synergism between mesenchymal stem cells and immunosuppressive drugs in a murine heart transplantation model. Transpl Immunol. 2011;25(23):141-147. doi:10.1016/j.trim.2011.06.002 
40. Jia Z, Jiao C, Zhao S, et al. Immunomodulatory effects of mesenchymal stem cells in a rat corneal allograft rejection model. Exp Eye Res. 2012;102:44-49. doi:10.1016/j.exer.2012.06.008

41. Zhang W, Qin C, Zhou ZM, et al. Mesenchymal stem cells modulate immune responses combined with cyclosporine in a rat renal transplantation model. Transplant Proc. 2007;39(10):3404-3408. doi:10.1016/j.transproceed.2007.06.092

42. Zhang Q, Shi S, Liu Y, et al. Mesenchymal stem cells derived from human gingiva are capable of immunomodulatory functions and ameliorate inflammation-related tissue destruction in experimental colitis. J Immunol. 2009;183(12):7787-7798. doi:10.4049/ jimmunol.0902318

43. Coccè V, Farronato D, Brini AT, et al. Drug loaded gingival mesenchymal stromal cells (GinPa-MSCs) inhibit in vitro proliferation of oral squamous cell carcinoma. Sci Rep. 2017;7:9376.

44. Górski B. Gingiva as a new and the most accessible source of mesenchymal stem cells from the oral cavity to be used in regenerative therapies. Postepy Hig Med Dosw. 2016;70:858-871. doi:10.5604/17322693.1214383

45. Fournier BP, Larjava H, Häkkinen L. Gingiva as a source of stem cells with therapeutic potential. Stem Cells Dev. 2013;22:3157-3177. doi: $10.1089 / \mathrm{scd} .2013 .0015$

46. Kim D, Lee AE, Xu Q, et al. Gingiva-derived mesenchymal stem cells: potential application in tissue engineering and regenerative medicine-a comprehensive review. Front Immunol. 2021;12:667221. doi:10.3389/fimmu.2021.667221

47. Noda A, Kinoshita K, Sakurai A, et al. Hyperglycemia and lipopolysaccharide decrease depression effect of interleukin 8 production by hypothermia: an experimental study with endothelial cells. Intensive Care Med. 2008;34(1):109-115. doi:10.1007/s00134-007-0861-2

48. Hagiwara S, Iwasaka H, Hasegawa A, et al. Hyperglycemia contributes to cardiac dysfunction in a lipopolysaccharide-induced systemic inflammation model. Crit Care Med. 2009;37(7):2223-2227. doi:10.1097/CCM.0b013e3181a007c6

49. Raetzsch CF, Brooks NL, Alderman JM, et al. Lipopolysaccharide inhibition of glucose production through the Toll-like receptor-4, myeloid differentiation factor 88, and nuclear factor kappa b pathway. Hepatology. 2009;50(2):592-600. doi:10.1002/hep.22999
50. Tweedell A, Mulligan KX, Martel JE, et al. Metabolic response to endotoxin in vivo in the conscious mouse: role of interleukin-6. Metabolism. 2011;60(1):92-98. doi:10.1016/j.metabol.2009.12.022

51. Hagar JA, Edin ML, Lih FB, et al. Lipopolysaccharide potentiates insulin-driven hypoglycemic shock. $J$ Immunol. 2017;199 (10):3634-3643. doi:10.4049/jimmunol.1700820

52. Płóciennikowska A, Hromada-Judycka A, Borzęcka K, et al. Cooperation of TLR4 and raft proteins in LPS-induced pro-inflammatory signaling. Cell Mol Life Sci. 2015;72(3):557-581. doi:10.1007/s00018-014-1762-5

53. Zamyatina A, Heine H. Lipopolysaccharide recognition in the crossroads of TLR4 and caspase-4/11 mediated inflammatory pathways. Front Immunol. 2020;11:585146. doi:10.3389/fimmu.2020.585146

54. Meng L, Li L, Lu S, et al. The protective effect of dexmedetomidine on LPS-induced acute lung injury through the HMGB1-mediated TLR4/NF- $\mathrm{KB}$ and PI3K/Akt/mTOR pathways. Mol Immunol. 2018;94:7-17. doi:10.1016/j.molimm.2017.12.008

55. Liu CH, Zhang WD, Wang JJ, Feng SD. Senegenin ameliorate acute lung injury through reduction of oxidative stress and inhibition of inflammation in cecal ligation and puncture-induced sepsis rats. Inflammation. 2016;39(2):900-906. doi:10.1007/s10753-016-0322-6

56. Stortz JA, Raymond SL, Mira JC, et al. Murine models of sepsis and trauma: can we bridge the gap? ILAR J. 2017;58(1):90-105. doi:10.1093/ilar/ilx007

57. Seemann S, Zohles F, Lupp A, et al. Comprehensive comparison of three different animal models for systemic inflammation. Comparative Study. J Biomed Sci. 2017;24(1):60. doi:10.1186/ s12929-017-0370-8

58. Recknagel P, Gonnert FA, Halilbasic E, et al. Mechanisms and functional consequences of liver failure substantially differ between endotoxaemia and faecal peritonitis in rats. Liver Int. 2013;33:283-293. doi:10.1111/liv.12012

59. Deitch EA. Animal models of sepsis and shock: a review and lessons learned. Shock. 1998;9:1-11. doi:10.1097/00024382-19980100000001
Infection and Drug Resistance

\section{Publish your work in this journal}

Infection and Drug Resistance is an international, peer-reviewed openaccess journal that focuses on the optimal treatment of infection (bacterial, fungal and viral) and the development and institution of preventive strategies to minimize the development and spread of resistance. The journal is specifically concerned with the epidemiology of
Dovepress

antibiotic resistance and the mechanisms of resistance development and diffusion in both hospitals and the community. The manuscript management system is completely online and includes a very quick and fair peerreview system, which is all easy to use. Visit http://www.dovepress.com/ testimonials.php to read real quotes from published authors. 